\title{
A Machine Learning Approach to Detect Wounded Dog
}

\author{
Amit Lal Das \\ Department of CSE, \\ Sylhet International University, \\ Shamimabad, Bagbari, Sylhet, \\ Bangladesh
}

\author{
K.M. Ashikur Rahman \\ Department of CSE, \\ Sylhet International University, \\ Shamimabad, Bagbari, Sylhet, \\ Bangladesh
}

\begin{abstract}
Now, everything is automated. There are various kinds of animals around us. Some animals live with us, some have residence, some have an owner, some are in a specific area. Dogs are such kind of animal that they live with us. But many of the dogs have no owner or residence. And they cannot have a healthy life. They also get wounds in many ways but have no remedy. They live with us or around us. If they are wounded, the disease can spread by them. So we need to detect them immediately and concern the authority. So we make such kind of system which can identify a wounded dog. We use a dataset of 409 data for this purpose in yolov3.
\end{abstract}

\section{Keywords}

Yolov3, Object detection, Machine Learning, Wounds, Dog, Google colab.

\section{INTRODUCTION}

Peoples are fond of dogs to keep with them. But all animals have no shelters. They are around us. They have also diseases. But if they are effects, they need to cure up. Otherwise, they may spread the disease. They can injure in many ways. But they need to detect fast.

To get rid of this problem, we need an automated solution. And we make a system using machine learning yolov3 to solve this problem. It can fast detect the dog is injured or not. We used a dataset of 645 images. We annotate all the data. We used YOLOv3 deep learning to train the model with the help of Google colab.

Our target is to identify, bite, burns, cavities in the body, skin cut by something, bullet infect, and accidental injury.

\section{RELATED WORKS}

We saw the previous result related to this research. A current advanced site like Research gate, IEEE, and Google Scholar is used for that research literature review.

We saw much of the literature, all the researchers have tried to find the physical disease of dog, also used radar to detect a specific animal like a dog. [5][6][7][8][9][10][11][12][13][14][15][16]

For animals, we found no literature like a wounded animal detection. All that we found is just animal detection. Researchers tried to detect an animal by different methodology. [17]

But we got no related work like deep machine learning to identify wounded dogs. And we want to build this method.

In our research, we made a solution to detect wounded dogs. Different kinds of wounds we identify using our research approach. It helps to detect a wounded dog easily.

\section{METHODOLOGY}

\subsection{Datset Prepare}

We collected 409 images of a dataset for this. YOLOv3 was used to train the dataset in Google collaborate and test the data for this system.

We have created a dataset for our research. This dataset contains 409 images. Which has trained from google colab.[4]

We collected data from a different source. Some of them have collected from real life. Some of them have been collected from google(licensable), and Some of them from facebook.

The average size of images that we used is $9.58 \mathrm{~KB}$. The average horizontal resolution of images that we used is $96 \mathrm{dpi}$. The average vertical resolution of images that we used is 96 dpi. The average dimensions of images that we used is $206 \mathrm{x}$ 206 pixel.

Let's see some example of Annotated wounded dog data given below in figure-1,
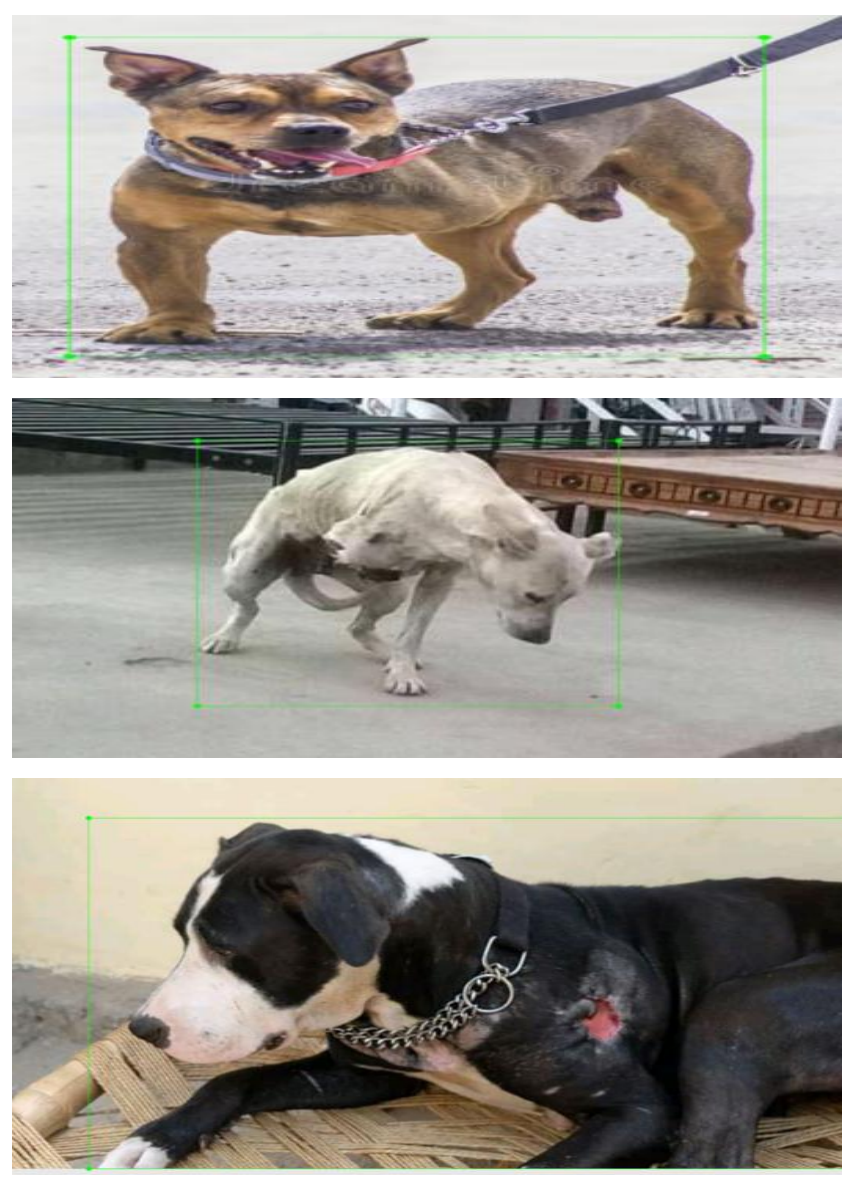

Figure-1: Images for Yolov3 Training 


\subsection{Yolov3 Working Procedure}

YOLO - You Only Look Once, it applies a single forward pass neural network to the whole image and predicts the bounding boxes and their class probabilities as well.

The YOLOv3 network divides an input image into $S \times 5$ grid of cells and predicts bounding boxes as well as class probabilities for each grid. Each grid cell is responsible for predicting $B$ bounding boxes and $C$ class probabilities of objects whose centers fall inside the grid cell. Bounding boxes are the regions of interest (ROI) of the candidate objects. The " $B$ " is associated with the number of using anchors. Each bounding box has $(5+C)$ attributes. The value of " 5 " is related to 5 bounding box attributes, those are center coordinates $\left(\mathrm{b}_{\mathrm{x}}, \mathrm{b}_{\mathrm{y}}\right)$ and shape $\left(\mathrm{b}_{\mathrm{h}}, \mathrm{b}_{\mathrm{w}}\right)$ of the bounding box, and one confidence score. The " $C$ " is the number of classes. The confidence score reflects how confidence a box contains an object.[1]

YOLOv3 network Architecture is shown in figure-2.

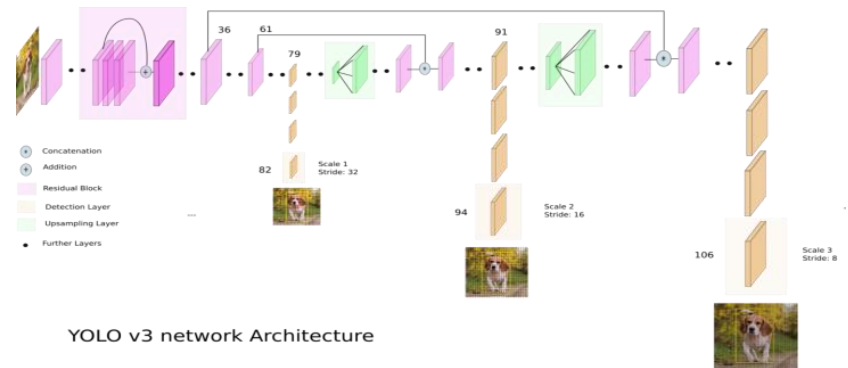

Figure-2: YOLOv3 network Architecture [1]

Let us consider an example below, where the input image is $416 \times 416$, and stride of the network is 32 . As pointed earlier, the dimensions of the feature map will be $13 \times 13$. We then divide the input image into $13 \times 13$ cells. Then, the cell (on the input image) containing the center of the ground truth box of an object is chosen to be the one responsible for predicting the object. In the image, it is the cell which marked red, which contains the center of the ground truth box (marked yellow).[2]

Gridding of the image is shown in figure-3. And features map at different scales is shown in figure-4.

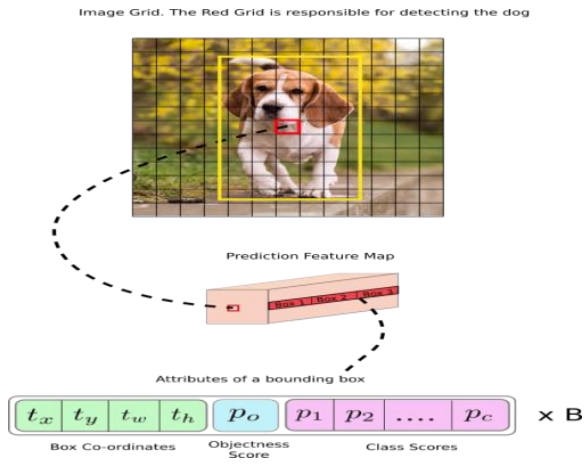

Figure-3: Image Grid [2]
Prediction Feature Maps at different Scales

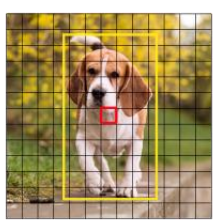

$13 \times 13$

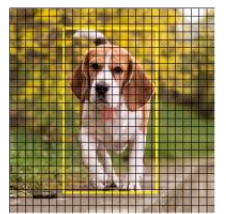

$26 \times 26$

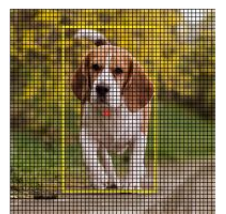

$52 \times 52$

Figure-4: Features map at different scales [2]

\subsection{Hardware and Software}

We have performed our experiment on a advanced platform.

NVIDIA-SMI 460.56, Driver Version: 460.32.03,CUDA

Version: 11.2, GPU Name Persistence-M Fan Temp

Perf Pwr:Usage/Cap, Bus-Id Disp.A Memory-Usage, Volatile Uncorr. ECC GPU-Util Compute M. MIG M. 0 Tesla T4 Off $\mid$ N/A $36 \mathrm{C}$ P8 $9 \mathrm{~W} / 70 \mathrm{~W}$, 00000000:00:04.0 Off 0MiB / 15109MiB, 0 0\% Default N/A, Processes:

GPU GI CI PID Type Process name GPU Memory
ID ID
Usage

CPU: Model name: Intel(R) Xeon(R) CPU @ 2.30GHz, Address sizes: 46 bits physical, 48 bits virtual, GPU: single 12GB NVIDIA Tesla K80 GPU, 13 GB RAM, Cloud TPU with 180 teraflops of computation, Intel Xeon processor with two core@2.30 GHz, !3 GB RAM

We annotate our dataset from labelImg platform.

\subsection{Training}

First, we annotated our data from the labelimg tool. Then we trained our dataset from google colab using the yolov3 model. Our dataset contains 409 data. Along with that, it has short details of the annotated area. And we prepare that dataset for training. Which can extend further.[4]

\subsection{Results and Analysis}

We are to show our test data results from the dataset we trained. For that result showing we are using 50 images of wounded dogs, five good dogs, one zebra, one deer, two airplanes, one tortoise, in a total of 60 images. As our dataset model trained using the YOLOv3 model, we test all the data separately. We settled our confidence label at 0.5 and our threshold label at 0.3 . 
Table-1: Validating the test data comparing with YOLOv3 model.

\begin{tabular}{cccc}
\hline $\begin{array}{c}\text { True } \\
\text { Positive }\end{array}$ & $\begin{array}{c}\text { True } \\
\text { Negative }\end{array}$ & $\begin{array}{c}\text { False } \\
\text { Positive }\end{array}$ & $\begin{array}{c}\text { False } \\
\text { Negative }\end{array}$ \\
\hline 46 cases & 7 cases & 4 cases & 3 cases \\
\hline
\end{tabular}

All the cases are shown in chart-1 in tabular form. On the left side, 0-50 indicates the case. On the right side below, TP means true positive, TN means, true negative, FP means false positive, FN means false positive.

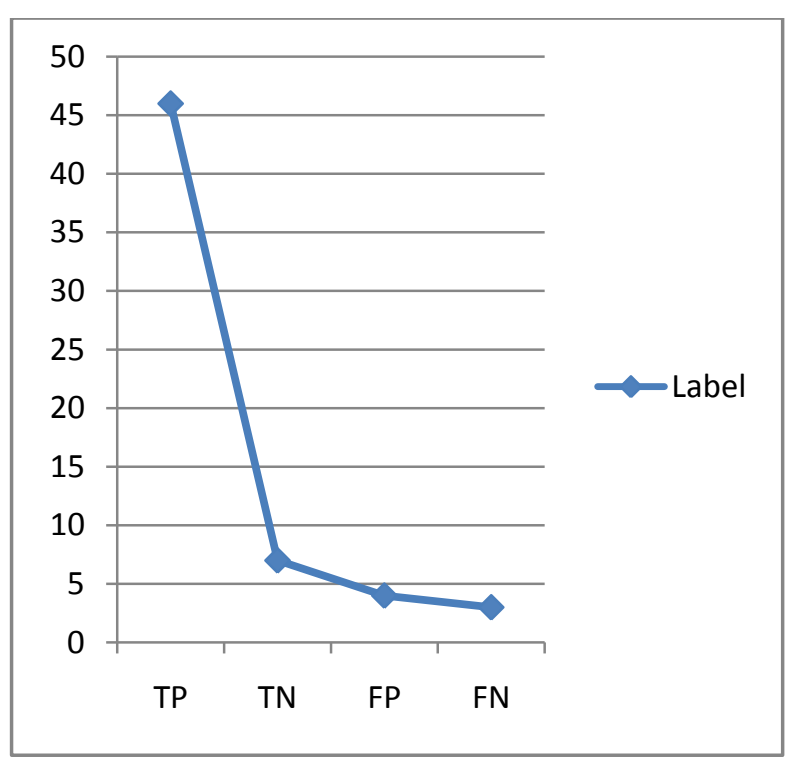

Chart 1: Confusion Matrix Representation

In predictive analytics, a table of confusion (sometimes also called a confusion matrix) is a table with two rows and two columns that reports the number of false positives, false negatives, true positives, and true negatives. This allows more detailed analysis than mere proportion of correct classifications (accuracy). Accuracy will yield misleading results if the data set is unbalanced; that is, when the numbers of observations in different classes vary greatly.[3]

The confusion matrix formula and equations are shown in figure-5.

\begin{tabular}{|c|c|c|}
\multicolumn{3}{|c}{ Confusion Matrix } \\
$\begin{array}{c}\text { Machual } \\
\text { counting } \\
\text { learning }\end{array}$ & True & False \\
\hline True & $\begin{array}{c}\text { True Positive } \\
\text { (TP) }\end{array}$ & $\begin{array}{c}\text { False Positive } \\
\text { (FP) }\end{array}$ \\
\hline False & $\begin{array}{c}\text { False Negative } \\
\text { (FN) }\end{array}$ & $\begin{array}{c}\text { True Negative } \\
\text { (TN) }\end{array}$ \\
\hline
\end{tabular}

\section{Equations:}

False positive rate( $(F P R)=F P /(F P+T N)$

False negative rate(FNR) $=\mathrm{FN} /(\mathrm{FN}+\mathrm{TP})$

Sensitivity $=\mathrm{TP} /(\mathrm{TP}+\mathrm{FN})$

Specificity $=T N /(T N+F P)$

Accuracy $=(\mathrm{TP}+\mathrm{TN}) /(\mathrm{TP}+\mathrm{TN}+\mathrm{FP}+\mathrm{FN})$

Youden index $=$ Sensitivity + Specificity -1

Figure-5: confusion matrix
According to the above formula,

TP is $46, \mathbf{T N}$ is $7, \mathbf{F P}$ is $4, \mathbf{F N}$ is 3 , and from the formula, with these values, we can evaluate every possible rate.

$\mathbf{F P R}=\quad 0.36, \mathbf{F N R}=\quad 0.0612, \quad$ Sensitivity $=$ 0.86792 , Specificity $=0.6363$ and Accuracy is $=0.8833$. This is around $\mathbf{8 8 . 3 3} \%$ of total average prediction.

FPR, FNR, Sensitivity, Specificity is rounded up and formatted in decimal. On the left side, $0-100$ is the accuracy rate. On the right side below, FPR, FNR, Sensitivity, Specificity is shown in percentage. This is shown in chart 2.

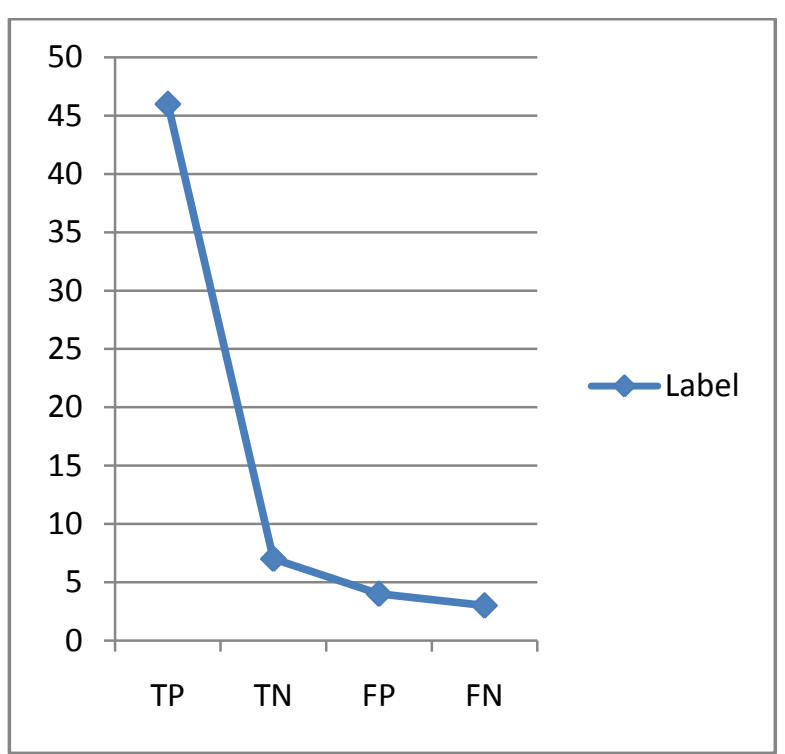

Chart 2: According to table of confusion matrix

Separately we tested 30 data to find the accuracy. In this case, we find out the accuracy rate in every test. The average accuracy is $76.825 \%$.

[19]

The difference between the two accuracy rates is shown in chart 3 . Here confusion matrix accuracy is the accuracy we find it out by using a confusion matrix. Separate accuracy is the accuracy we find it out from separately tested 33 data. On the left side, 0-90 is the accuracy rate. On the right side below, there plotted confusion matrix and separate accuracy in percentage.

\section{Rate}

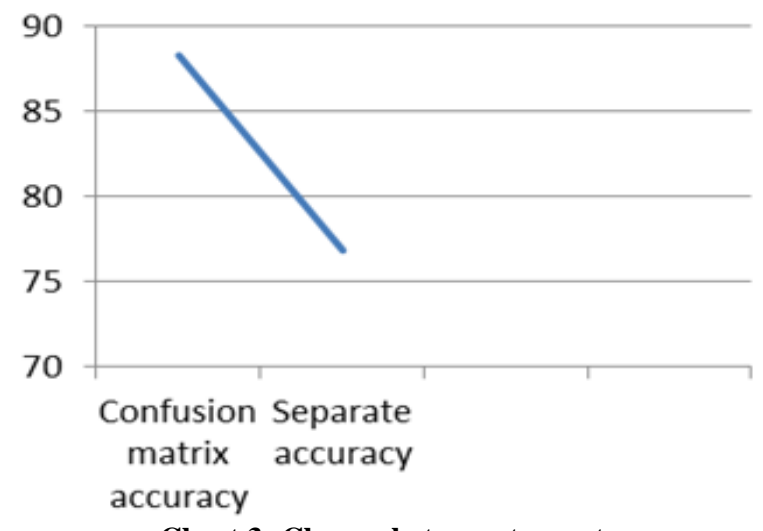

Chart 3: Change between two rates 
Here are some examples of test data.

These images were not taken from the training dataset.

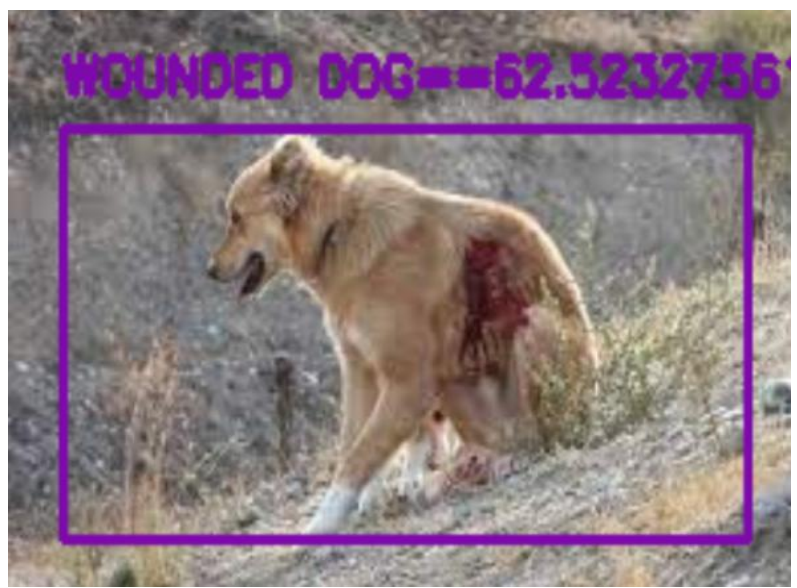

Figure-6: Images prediction, Wounded 62.52\%, Confidence 0.5, Threshold 0.3

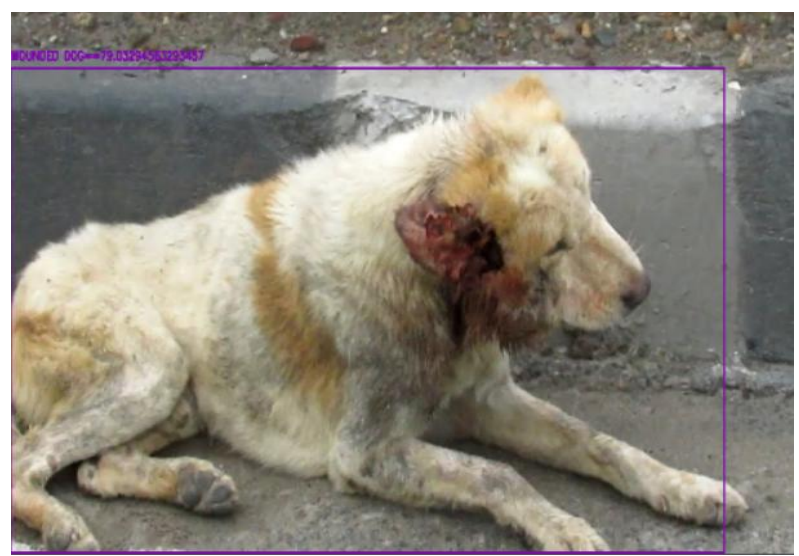

Figure-7: Images prediction, Wounded $79.02 \%$, Confidence 0.5, Threshold 0.3

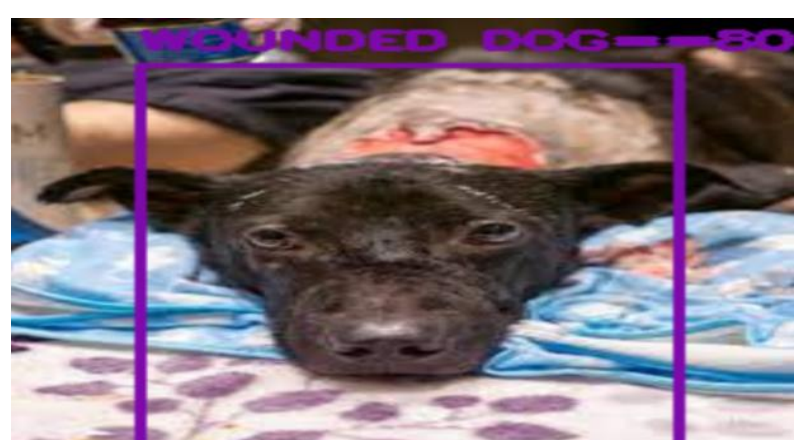

Figure-8: Images prediction, Wounded $80 \%$, Confidence 0.5 , Threshold 0.3

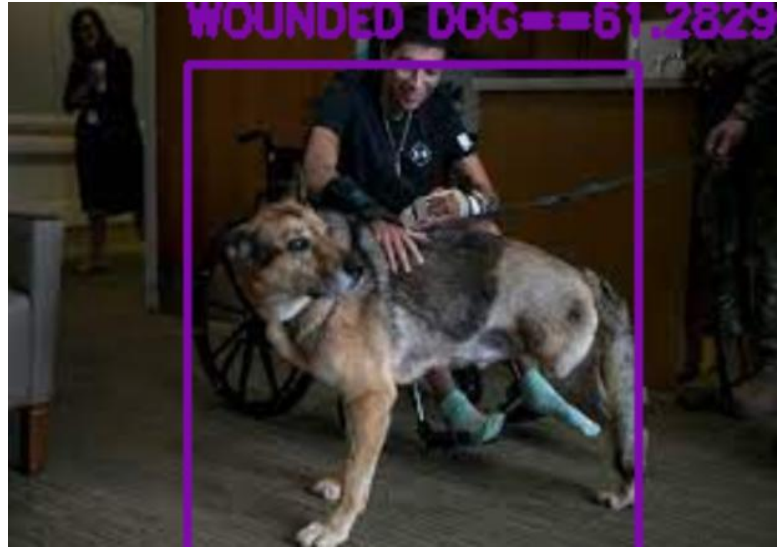

Figure-9: Images prediction, Wounded 61.28\%, Confidence 0.5, Threshold 0.3

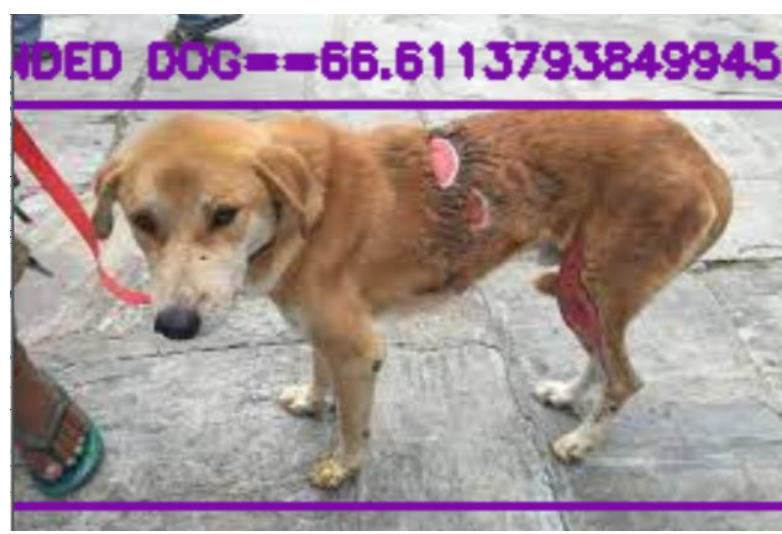

Figure-8: Images prediction, Wounded $66.61 \%$, Confidence 0.5, Threshold 0.3

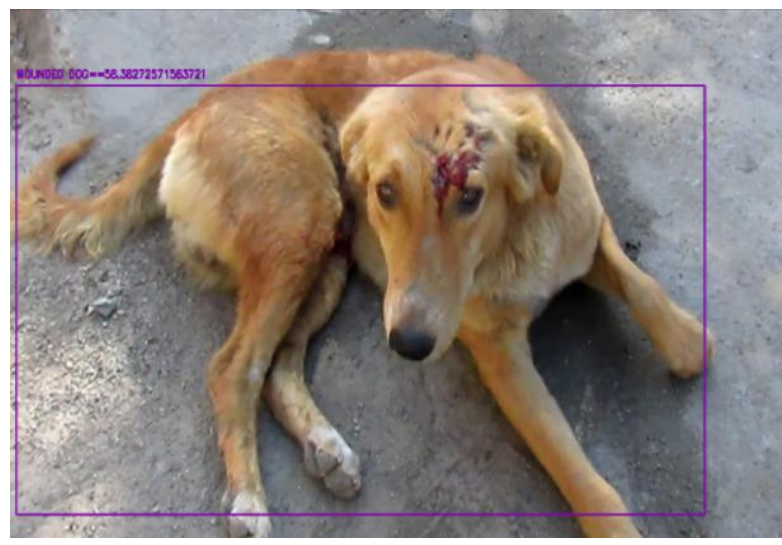

Figure-9: Images prediction, Wounded $58.38 \%$, Confidence 0.5, Threshold 0.3

\section{CONCLUSION}

As we evaluated a result from our model we can say that this is a much better model give better output in detecting wounded dog. YOLOv3 model is an efficient tool for this system, although it's not giving expected output all the time we want. But it is a better approach. This model can used in a real environment with a large number of productive datasets for accuracy. 


\section{FUTURE PLANS}

We will try to increase our dataset as we can give a near 100 percent accuracy. We will try to train our dataset in such a way that we can detect it more rapidly. Automation system addition to inform proper authority. Detection capability from long distances will be tried to add. We will try to add low light intensity detection.

\section{LIMITATIONS}

The small size of a dataset may give the average results compared to the high-level size of the dataset. And our dataset is small in size. We will try to increase it later. Distance is a fact in detection. The short distance can give better results according to the system's capability. We tested our system from two feet distance. Light intensity sometimes affects it. We faced it sometimes. It can be recovered with an advanced system.

\section{REFERENCES}

[1] https://machinelearningspace.com/yolov3-tensorflow-2part-1/

[2] https://www.kdnuggets.com/2018/05/implement-yolov3-object-detector- pytorchpart-1.html

[3] https://en.wikipedia.org/wiki/Confusion_matrix

[4] https://drive.google.com/file/d/1J7_zpNXe_4rBSGwJ1kn $\mathrm{JIDbxtcO}$ BrLC/view?usp=sharing

[5] (PDF) A Comparison of Different Wound Planimetry Methods in Dogs. (n.d.). Retrieved May 21, 2021, from https://www.researchgate.net/publication/327307521_A_ Comparison_of_Different_Wound_Planimetry_Methods _in_Dogs

[6] (PDF) Application of a temporary intestinal storage device in a small intestine gunshot wound dog model. (n.d.). Retrieved May 21, 2021, from https://www.researchgate.net/publication/348642656_Ap plication_of_a_temporary_intestinal_storage_device_in_ a_small_intestine gunshot_wound_dog_model

[7] (PDF) MANAGEMENT OF A DOG BITE WOUND: A CASE REPORT. (n.d.). Retrieved May 21, 2021, from https://www.researchgate.net/publication/348096055_M ANAGEMENT_OF_A_DOG_BITE_WOUND_A_CAS E_REPORT

[8] (PDF) Management of Diffuse Necrotic Cutaneous Wound in a Dog. (n.d.). Retrieved May 21, 2021, from https://www.researchgate.net/publication/346652240_Ma nagement_of_Diffuse_Necrotic_Cutaneous_Wound_in_a _Dog

[9] Antônio, W. H. S., Da Silva, M., Miani, R. S., \& Souza, J. R. (2019). A Proposal of an Animal Detection System Using Machine Learning. Applied Artificial Intelligence, 33(13), 1093-1106. https://doi.org/10.1080/08839514.2019.1673993
[10] Hanson, R. R., \& Munsterman, A. S. (2016). Treatment of Burn Injuries, Gunshot Wounds, and Dog-Bite Wounds. In Equine Wound Management: Third Edition (pp. 476-489). Wiley Blackwell. https://doi.org/10.1002/9781118999219.ch20

[11] Iacopetti, I., Patruno, M., Melotti, L., Martinello, T., Bedin, S., Badon, T., Righetto, E. M., \& Perazzi, A. (2020). Autologous Platelet-Rich Plasma Enhances the Healing of Large Cutaneous Wounds in Dogs. Frontiers in Veterinary Science, https://doi.org/10.3389/fvets.2020.575449

[12] Kim, J., Kim, D., Kim, J., Seo, D., Hwang, H., Kim, Y., Chung, T., Lim, S., Lee, H., \& Kim, M. S. (2020). Case Report: Surgical Treatment of Severe Facial Wounds and Proptosis in a Dog Due to a Traffic Accident. Frontiers in Veterinary Science, 7 https://doi.org/10.3389/fvets.2020.548279

[13] Maraki, S., Kastanis, G., Stafylaki, D., Masunt, S., Kapsetakis, P., \& Scoulica, E. (2018). Pasteurella multocida wound infection transmitted by a pet dog. GERMS, 8(4), 214-217. https://doi.org/10.18683/germs.2018.1149

[14] Reddell, P., De Ridder, T. R., Morton, J. M., Jones, P. D., Campbell, J. E., Brown, G., Johannes, C. M. Schmidt, P. F., \& Gordon, V. (2021). Wound formation, wound size, and progression of wound healing after intratumoral treatment of mast cell tumors in dogs with tigilanol tiglate. Journal of Veterinary Internal Medicine, 35(1), 430-441. https://doi.org/10.1111/jvim.16009

[15] RÎMBU, C., HORHOGEA, C., COZMA, A., CRETU, C., GRECU, M., RUSU, R., \& GUGUIANU, E. (2020). Analysis of Bacteriological Infected Dog and Cat Bite Wounds in Veterinary Medical Staff. Bulletin of University of Agricultural Sciences and Veterinary Medicine Cluj-Napoca. Veterinary Medicine, 77(1), 43. https://doi.org/10.15835/buasvmcn-vm:2019.0038

[16] Swaim, S. F., Gillette, R. L., Sartin, E. A., Hinkle, S. H., \& Coolman, S. L. (2000). Effects of a hydrolyzed collagen dressing on the healing of open wounds dogs. American Journal of Veterinary Research, 61(12), 1574 1578. https://doi.org/10.2460/ajvr.2000.61.1574

[17] Yu. Smolentsev, S., A. Gracheva, O., S. Gasanov, A., R. Amirov, D., M. Mukhutdinova, D., R. Shageeva, A., M Zukhrabova, Z., V. Pozyabin, S., A.Kozlov, N., I. Shumakov, N., A. Bykovskaya, T., P. Tsiulina, E., \& R. Idrisova, R. (2019). Hematological and Immunological Blood Parameters in the Treatment of Infected Wounds in Dogs. Journal of Engineering and Applied Sciences, 14(24), 9806-9809. https://doi.org/10.36478/jeasci.2019.9806.9809

[18] https://drive.google.com/file/d/1vDjYUbXjUy6W_zefx_i $\mathrm{tS} 1 w L e e Y 7 y i H Z / v i e w$ ?usp=sharing 